\title{
Ongoing Consideration of Cavity Evaluation Devices in the Treatment of Breast Cancer
}

\author{
Dennis R. Holmes, MD, FACS \\ USC Norris Comprehensive Cancer Center - Breast Surgery, Los Angeles, CA
}

The growing popularity of brachytherapy has greatly increased the use of single-entry balloon catheter devices for delivery of accelerated partial breast radiotherapy (APBI). To facilitate delayed, postoperative, percutaneous insertion of single-entry brachytherapy catheters, many surgeons elect to place temporary, expandable cavity evaluation devices (CED) at the time of lumpectomy to preserve the surgical cavity and maintain a tract between the skin and surgical cavity. ${ }^{1}$ At the time of brachytherapy catheter placement, the CED may be deflated, withdrawn from the cavity, and easily replaced by a single-entry brachytherapy catheter inserted through the very same tract.

In their article, "Spacer Balloons prior to Partial Breast Irradiation: Helpful or Hurtful?", Drs. Kuske and Zannis provide an excellent summary of the potential limitations and challenges associated with CED usage. ${ }^{2}$ While they have raised several important concerns, the authors' emphasis on the potential negative aspects of CED placement misses the opportunity to provide sufficient guidance on how and when they may be properly used.

Among the important concerns raised by the authors is the tendency of some surgeons to obtain multidisciplinary consultation only after CED placement. Preoperative discussion of the patient's treatment options in a multidisciplinary setting or referring the patient to a radiation oncologist prior to surgery will provide the best opportunity for the patient and her oncologists to identify and fully discuss the options of lumpectomy, APBI, CED usage, and the implications of unsuitable pathology. This process not only helps to identify patients who are suboptimal candidates for APBI, but also allows coordination

(C) Society of Surgical Oncology 2011

Published Online: 26 April 2011

D. R. Holmes, MD, FACS

e-mail: DHolmes@usc.edu of surgery, catheter exchange, and radiation therapy planning to avoid the significant delays in the initiation of APBI that the authors identify as being contributory to devicerelated infections. Preoperative consultation also gives the patient the opportunity to discuss their potential participation in clinical trials that might be impacted by CED placement.

Another compelling issue raised by the authors is the higher incidence of wound infection associated with intraoperative placement of a brachytherapy catheter (the open-cavity technique) compared with postoperative, percutaneous brachytherapy catheter placement (the closedcavity technique). ${ }^{3}$ However, the association between CED placement and infection is far from clear. In referencing the 2-year outcome data of the MammoSite Registry, the authors acknowledge that CED usage was not specifically addressed in the publication. Although it is reasonable for the authors to infer that intraoperative placement of a CED is analogous to open-cavity placement of a brachytherapy catheter in terms of its risk of infection, the results of the MammoSite Registry are confounded by the lack of clear guidelines regarding the use of antibiotics. In fact, only $71 \%$ of MammoSite Registry participants received antibiotics. Perhaps patients who had catheters placed intraoperatively were less likely to be prescribed oral antibiotics because they were routinely given a single dose of intravenous antibiotics preoperatively. Alternatively, it could be that patients who had catheters placed postoperatively were more likely to be prescribed postprocedural oral antibiotics because they did not receive the benefit of preprocedural intravenous antibiotics? The MammoSite Registry update does not elucidate these factors. Furthermore, since there is a finite risk $(1-4 \%)$ of surgical-site infection after breast-conserving surgery, to what degree does the higher incidence of infection after the open-cavity technique reflect the combined result of postlumpectomy infection and catheter-related infection. ${ }^{3,4}$ Interestingly, the 
MammoSite Registry reported a higher overall incidence of acute skin toxicity compared to breast infection (12 versus $9 \%$ ) and a lower incidence of acute skin toxicity with antibiotic usage $(P<0.0001){ }^{3}$ This suggests that postprocedural oral antibiotics should be routinely given to patients undergoing catheter-based APBI regardless of the timing of catheter placement.

Another issue raised by Drs. Kuske and Zannis is the wasted cost of a CED if it must be removed and discarded because of unfavorable surgical pathology. ${ }^{2}$ While we must remain vigilant about controlling health care costs, the actual cost of a CED (approximately US \$150) is but a fraction of the cost of the brachytherapy catheter (US $\$ 2,800$ ) or the hospital costs of margin re-excision or completion axillary lymph node dissection (ALND). Nonetheless, it is incumbent upon surgeons to make their very best effort to understand the extent of disease within the breast and axilla prior to performing lumpectomy and axillary staging in APBI candidates regardless of whether or not CED placement is planned. Evidence that this is achievable can be found in the recently published prospective, randomized, controlled trial (the TARGIT trial) comparing single-fraction intraoperative APBI administered at the time of lumpectomy versus conventional postoperative whole-breast external-beam radiotherapy. ${ }^{5}$ In both treatment arms, surgeons were able to achieve negative surgical margins in $90 \%$ of participants, with fewer than $15 \%$ of participants requiring additional therapy (e.g., whole-breast external-beam radiotherapy) due to more extensive disease (e.g., extensive intraductal component or positive nodes). Similar success should be achievable by high-volume breast surgeons, and should result in relatively few patients requiring CED removal and disposal.

Multiple strategies may be utilized to optimize preoperative and intraoperative evaluation of disease extent. Preoperative strategies include breast magnetic resonance imaging (MRI) and axillary ultrasound, with minimally invasive biopsy of any additional abnormal imaging findings. Intraoperatively, the surgeon may use multiple wire localization, intraoperative ultrasound, specimen radiography, and intraoperative margins assessment (gross, frozen section, or touch prep) to increase the probability of successful lumpectomy. A surgeon can elect to forgo CED placement in a patient whose imaging or minimally invasive biopsy findings (e.g., an extensive intraductal component) identifying the patient as a poor candidate for APBI or CED placement. Using selection criteria similar to these, McCready et al. were able to achieve a $98 \%$ negative margin rate following lumpectomy among older women (age $>50$ years) with ultrasound-visible, wire-localized, core needle biopsy-proven breast cancer. ${ }^{6}$ These features are also consistent with the American Society of
Therapeutic Radiation Oncology's recommended guidelines for patients undergoing APBI. ${ }^{7}$

Despite their potential shortcomings, CEDs do provide some technical advantages to APBI. In additional to maintaining the surgical cavity, CEDs allow the user to accurately measure the tissue thickness overlying the CED prior to brachytherapy catheter placement to ensure that the skin spacing exceeds the $6 \mathrm{~mm}$ minimum thickness recommended for catheter-based brachytherapy. In the MammoSite Registry, skin spacing $<6 \mathrm{~mm}$ was associated with an increased risk of acute skin toxicity and telangiectasias. $^{3}$ Insufficient skin spacing discovered after brachytherapy catheter placement may require removal and disposal of a relatively expensive brachytherapy catheter. The CED may also be used to dilate the catheter tract to facilitate brachytherapy catheter insertion. This is accomplished by alternately inflating and deflating the CED with a few milliliters of saline as the CED is being drawn from the surgical cavity toward the skin. Dilatation of the catheter tract in this manner permits the brachytherapy catheter to be easily introduced into the surgical cavity without resistance, which saves money by saving time. CED placement also minimizes the need for a trocar, which lowers the risk of pneumothorax or vessel laceration.

One additional application of the CED should also be considered. In recent years, a variety of single-entry, expandable, balloon catheter brachytherapy systems have been introduced for delivery of APBI. Both the Intrabeam balloon catheter (Carl Zeiss-Meditec, Inc., Oberkochen, Germany) and the Axxent balloon catheters (Xoft Inc., Sunnyvale, CA) utilize multiple, fixed-diameter, expandable brachytherapy catheters ranging in diameter from 3 to $5 \mathrm{~cm}$ and 3 to $6 \mathrm{~cm}$, respectively. Unlike most single-entry catheters that may individually be inflated to variable diameters, the Intrabeam and Axxent catheters require that the user select among several fixed-diameter catheters to choose the one that best fits the lumpectomy cavity. Catheter selection is aided by ultrasound assessment of surgical cavity dimensions, but surgical cavity dimensions can be more accurately measured using a fluid-filled CED to estimate cavity volume and cavity configuration.

In summary, Drs. Kuske and Zannis have drawn attention to several very important issues raised by CED placement. $^{2}$ However, most of these concerns are not without remedy, and there are indeed several important advantages to using a CED in APBI. Most importantly, surgeons offering APBI should extend to their patients the benefit of a preoperative consultation with a radiation oncologist to ensure that their patients fully understand their radiotherapy options as well as the implications of unfavorable pathology and CED placement. The small minority of patients with unfavorable surgical pathology might indeed be disappointed by CED removal, but they 
will likely be considerably more disappointed by the implications of the surgical pathology, namely the need to undergo additional surgery, standard whole-breast radiotherapy, or both.

\section{REFERENCES}

1. Beitsch PD, Hodge CW, Dowlat K, Francescatti D, Gittleman MA, Israel $\mathrm{P}$, et al. The surgeon's role in breast brachytherapy. Breast $J$. 2009; 15(1):93-100.

2. Kuske R, Zannis V. Spacer balloons prior to partial breast irradiation: helpful or hurtful? Ann Surg Oncol. 2011;18. doi: 10.1245/s10434-011-1682-7.

3. Cuttino LW, Keisch M, Jenrette JM, Dragun AE, Prestidge BR, Quiet CA, et al. Multi-institutional experience using the MammoSite radiation therapy system in the treatment of early-stage breast cancer: 2-year results. Int J Radiat Oncol Biol Phys. 2008;71(1): $107-14$.
4. Hall JC, Willsher PC, Hall JL. Randomized clinical trial of singledose antibiotic prophylaxis for non-reconstructive breast surgery. Br J Surg. 2006;93:1342-6.

5. Vaidya JS, Joseph DJ, Tobias JS, Bulsara M, Wenz F, Saunders C, et al. Targeted intraoperative radiotherapy versus whole breast radiotherapy for breast cancer (TARGIT-A trial): an international, prospective, randomised, non-inferiority phase 3 trial. Lancet. 2010;376(9735):91-102.

6. Schiller D, Le L, Cho B, Youngson B, McCready D. Factors associated with negative margins of lumpectomy specimen: Potential use in selecting patients for intraoperative radiotherapy. Ann Surg Oncol. 2008;15(3):833-42.

7. Smith BD, Arthur DW, Buchholz TA, Haffty BG, Hahn CA, Hardenbergh $\mathrm{PH}$, et al. Accelerated partial breast irradiation consensus statement from the American Society for Radiation Oncology (ASTRO). Int J Radiat Oncol Biol Phys. 2009;74(4): 987-1001. 